\title{
The Role of Telomere Dysfunction in Driving Genomic Instability
}

The mechanistic role of radiation-induced genomic instability in radiation carcinogenesis is an attractive hypothesis that remains to be rigorously tested. There are few in vivo studies on which to base judgments, but work in our laboratory with mouse models of radiogenic mammary neoplasia provided the first indications that certain forms of genetically predisposed radiation-induced genomic instability may contribute to tumor development. The central goal of this research project is to more firmly establish the mechanistic basis of this radiation-associated genomic instability and, from this, to assess whether such induced instability might play a major role in tumorigenesis at low doses of low LET radiation. In the case of mouse mammary tumors, susceptibility to induced instability is expressed as an autosomal recessive trait in mammary epithelial cells and is manifest largely as excess chromatid damage. Recently published studies associate this form of instability with DNA repair deficiency, polymorphic variation in the gene encoding DNA-PKcs (Prkdc), and mammary associated susceptibility. The underlying hypothesis being tested in this project is that tumorassociated genomic instability is preferentially expressed in certain recombinogenic genomic domains and that these may be cell lineage/individual-specific.

Our studies have focused on the induction of telomere dysfunction following exposure to ionizing radiation and the role of DNA-PKcs in this process. Telomeres consist of tandem arrays of short, repetitive G-rich sequence bound by a variety of telomere-associated proteins that together form a dynamic terminal structure that "caps" the ends of linear chromosomes, providing protection from illegitimate recombination, exonucleolytic attack and degradation. The cellular importance of functional telomeres is evidenced by the fact that they are essential for continuous cellular proliferation, an observation that has profound implications in our understanding of aging and cancer.

In striking contrast to natural chromosomal termini, broken chromosome ends produced by DNA doublestrand breaks (DSBs) are highly recombinogenic, and represent a major threat to the integrity of the cell's genome. As potent inducers of mutations and cell death, DSBs are arguably the most dangerous form of DNA damage. The correct repair of DSBs is essential for maintaining the genetic integrity of the cell, as erroneous repair can lead to chromosomal rearrangements such as translocations, which produce novel juxtapositions of DNA sequences at the exchange breakpoints. Cancer is frequently associated with such chromosomal abnormalities.

We have demonstrated that effective telomeric end capping of mammalian chromosomes unexpectedly requires proteins more commonly associated with DNA DSB repair. Ku70/Ku80 and DNA-PKcs all participate in DSB repair through non-homologous end joining (NHEJ). Mutations in any of these genes cause spontaneous chromosomal end-to-end fusions that maintain large blocks of telomeric sequence at the points of fusion. The fusions, which contribute significantly to the background level of chromosomal aberrations, are not a consequence of telomere shortening, nor are they telomere associations. We have also demonstrated that nascent telomeres produced via leading-strand DNA synthesis are especially susceptible to these end-to-end fusions, suggesting a crucial difference in postreplicative processing of telomeres that is linked to their mode of replication.

We have found that impaired end capping in DNA-PKcs-deficient genetic backgrounds not only allows dysfunctional telomeres to join to one another (telomere-telomere fusion), but also to broken chromosome ends created by radiation-induced DSBs (telomere-DSB fusion). In initial studies, DNA-PKcs-deficient cells from mice having the scid mutation were exposed to graded doses of $\gamma$-rays, a potent inducer of DSBs. The strand-specific molecular cytogenetic technique of Chromosome-Orientation Fluorescence in situ Hybridization (CO-FISH) was utilized to distinguish true telomere-DSB events from telomere-telomere fusions. Both types of end-joining events were observed, but only telomere-DSB fusions were induced by radiation, and this in a dose-dependent, linear fashion. Our results demonstrated for the first time that the radiation-sensitive phenotype of scid cells is not due solely to ineffective repair of DSB. Rather, telomereDSB misjoining provides an additional pathway for misrepair in scid cells that does not exist in repairproficient cells. These novel chromosomal rearrangements, which inappropriately maintain interstitial blocks of telomere sequence, are expected to have unusual properties whose consequences for the cell are not well understood. Interstitial telomere sequences have been shown to be a source of instability. Importantly, telomere-DSB fusion removes just one of the two ends created by a DSB, thereby rendering the remaining 
broken end capable of driving on-going chromosomal instability. It is also noteworthy that telomere-DSB fusions only require a single ionizing radiation-induced DSB, a process that will influence the shape of the dose response curve in unpredictable ways at low doses.

Our earlier work supported a link between partial deficiency of DNA-PKcs, impaired telomeric end capping and the radiogenic breast cancer-prone phenotype of Balb/c mice. Examination of mammary epithelial clones revealed telomere fusion events in a tumorigenic clone. Continued investigation utilizing the Balb/c mouse model has not only confirmed the presence of telomere-telomere fusion, but has also demonstrated a dose response for telomere-DSB fusion in BALB/c mouse mammary cells that rivals and even surpasses dicentric formation. Additionally, we now have evidence suggesting that following exposure to low doses of $\gamma$ radiation telomere fusion events in the Balb/c background of partial DNA-PKcs deficiency contribute to delayed instability in mouse mammary epithelial cells.

In order to identify chromosomes involved in Balb/c telomere-DSB fusions, as well as to characterize any clonal rearrangements, an essential aspect of demonstrating the oncogenic potential of these novel fusion events, we developed an approach to combine mouse SKY (Spectral Karyotyping) with telomere CO-FISH (termed SKYCO-FISH). Preliminary comparisons of side-by-side images revealed a clonal translocation (8:12) possessing a CO-FISH telomere-DSB signal/pattern at the translocation breakpoint. Another approach we are using to determine loci involved in instability and tumorigenesis in mouse mammary cells has been to analyze radiation-altered cells using BAC-CGH array technology. Comparison of Balb/c normal mammary vs. mammary tumor DNA revealed an amplification on chromosome 11 (BAC D11MIT253) that corresponds to a region previously identified by Ron DePinho's group as recurring in mouse adenocarcinomas, and additionally has synteny to human $17 q 25.1$, a region frequently amplified in breast carcinoma.

We continue characterization of dysfunctional telomeres in DNA-PKcs deficient backgrounds, including BALB/c, and have additional evidence supporting our model that these uncapped telomeres behave as DSBs, despite the presence of ample telomeric sequence. One particularly revealing approach has been to combine immunofluorescence detection of the damage response factor $\gamma$-H2AX with telomere FISH. In agreement with our molecular cytogenetic analyses, co-localization of $\gamma$-H2AX foci and telomere signals in S-phase interphase nuclei demonstrates that telomeres are experiencing end-capping failure following replication and are triggering a $\gamma$-H2AX DSB damage response. We have previously demonstrated that the kinase activity of DNA-PKcs is required for effective telomeric end-capping function. Utilizing another specific inhibitor of DNA-PKcs kinase activity in Ligase IV-/- mouse cells, we now confirm that telomere fusion is mediated by NHEJ; i.e., they require LigIV. These studies give mechanistic insight, but they also suggest that the telomere phenotype of DNA-PK deficiency is underestimated since the pathway responsible for telomere fusion formation is the same one that creates the dysfunction.

Most recently, we've explored the telomeric roles of newly identified DNA-PKcs auto-phosphorylation sites/clusters. We find that auto-phosphorylation of Thr-2609, but not Ser-2056, is an important in vivo target for DNA-PKcs end-capping function at mammalian telomeres. Our results fit the proposed reciprocal action of these two autophosphorylation sites, separating function of end-processing vs. end-joining. They also suggest a possible mechanism of DNA-PKcs action at telomeres and therefore, in preserving genomic stability.

Thus, impaired telomere function, as a significant source of spontaneous and radiation-induced chromosomal instability, has the potential to contribute to the cancer-prone phenotype associated with even partial DSB repair deficiency. Beyond their established role in maintaining the lengths of terminal sequences, telomeres have additional critical capping functions that influence both chromosomal radiosensitivity and preservation of genomic stability. Increasing our understanding of previously unrecognized relationships between the radiation/DNA damage response and telomere function will facilitate predicting the carcinogenic risk of exposure to low doses of ionizing radiation. 